\title{
Lesioni ipofisarie nel Carney Complex
}

\author{
Gianluca Occhi ${ }^{1} \cdot$ Daniela Regazzo $^{2} \cdot$ Mattia Barbot $^{2}$
}

Accettato: 11 giugno 2020 / Pubblicato online: 31 maggio 2021

(c) The Author(s) 2021

\section{Introduzione}

Il Carney Complex (CNC) è una rara sindrome ereditaria caratterizzata da pigmentazione cutanea anomala, mixomi cardiaci e cutanei, schwannomi e anomalie endocrine. Nel 70\% dei casi il CNC è trasmesso con modalità autosomica dominante a penetranza pressoché completa. Nel restante $30 \%$ dei casi questa condizione si manifesta in forma sporadica, spesso come risultato di mutazioni de novo. Caratterizzata da eterogeneità genetica, il CNC è dovuto nel $70 \%$ dei casi a mutazioni inattivanti del gene PRKARIA, un elemento del complesso multiproteico della protein chinasi A (PKA), centrale nella trasduzione del segnale cAMP.

L'iperattività endocrina può derivare da una serie di neoplasie, tra cui la displasia surrenalica nodulare pigmentata (PPNAD), adenomi ipofisari (AI), il tumore a grandi cellule del Sertoli calcifico e tumori tiroidei [1].

\section{Acromegalia}

L'ipersecrezione di GH è un evento comune nei pazienti con CNC e si traduce in elevati livelli sierici di GH e IGF-1 nel $75 \%$ dei casi. L'ipersecrezione di GH in questi pazienti, tuttavia, non è sempre indicativa di un AI rilevabile alla risonanza magnetica (RMN). L'acromegalia clinicamente evidente si osserva, infatti, in $\sim 10 \%$ dei casi CNC con imaging positivo per una lesione ipofisaria e rappresenta la prima manifestazione di malattia nel $7 \%$ dei casi [2]. Il par-

$凶$ G. Occhi

gianluca.occhi@unipd.it

1 Dipartimento di Biologia, Università degli Studi di Padova, Padova, Italia

2 U.O.C. Endocrinologia, Dipartimento di Medicina-DIMED, Azienda ospedaliera-Università di Padova, Padova, Italia

ticolare profilo ormonale dei pazienti $\mathrm{CNC}$ può dipendere da iperplasia ipofisaria, tipicamente delle cellule mammosomatotrope che, in alcuni casi, precede la formazione dell'AI. L'acromegalia biochimica può esser svelata dalla riposta anomala di GH a un test da carico orale di glucosio o dalla sua risposta paradossa al TRH test.

Sebbene simili a livello istopatologico a quelli sporadici, gli AI GH-secernenti (AI-GH) nel CNC hanno caratteristiche peculiari. È presente infatti, multifocalità, spesso una componente iperplastica, e positività ormonale multipla all'immunoistochimica. Quasi tutti gli AI-GH risultano positivi anche alla prolattina (PRL) - con ripercussione sul profilo biochimico - ma non è raro osservare positività anche per altri ormoni ipofisari ( $\alpha$-subunità, TSH- $\beta$, LH- $\beta$ e FSH- $\beta$ ) [3].

\section{Iperprolattinemia}

Sebbene quelle che coinvolgono l'asse GH/IGF-1 siano le più comuni, le anomalie ipofisarie nel $\mathrm{CNC}$ sembrano comportare anche un'anomala secrezione di PRL. È la componente ipofisaria mammo-somatotropa, infatti, la più interessata nei processi iperplastici o di trasformazione in questi pazienti. L'iperprolattinemia, sebbene quasi sempre modesta $(<100 \mathrm{ng} / \mathrm{ml})$, è stata osservata sia in pazienti CNC acromegalici $(\sim 25 \%)$ che in quelli privi di evidenti segni di acromegalia clinica e/o biochimica $(\sim 64 \%)$ [3]. L'iperprolattinemia franca associata ad adenomi ipofisari PRLsecernenti sembra essere, invece, una condizione piuttosto rara.

\section{Malattia di Cushing}

Nel CNC la sindrome di Cushing (CS) è comunemente associata a PPNAD; tuttavia, sebbene rare, sono state descritte 
forme ACTH-dipendenti di origine ipofisaria [4]. In presenza di valori di ACTH dosabili, l'origine ipofisaria dell'ipercortisolismo va confermata attraverso i test al CRH e al desametasone ad alte dosi. Inoltre, in caso di test discordanti e RMN ipofisaria negativa, il cateterismo dei seni petrosi consente di escludere la secrezione ectopica di ACTH - mai peraltro riportata nel $\mathrm{CNC}$ - ma soprattutto confermare la diagnosi di CS. Negli unici due casi riportati, la chirurgia trans-naso-sfenoidale si è rivelata risolutiva nel rimuovere la lesione ipofisaria che, all'esame istologico, è risultata un microadenoma ACTH positivo. Entrambi i casi presentavano una mutazione germinale inattivante in PRAKARIA e perdita di eterozigosi nella regione $17 \mathrm{q} 24$ a livello somatico, supportando il possibile ruolo patogenetico di queste mutazioni nella CS CNC-associata.

\section{Patogenesi}

Il ruolo delle mutazioni PRKARIA nella trasformazione neoplastica ipofisaria rimane da chiarire. Si ritiene tuttavia che, analogamente agli AI-GH sporadici mutati nell'oncogene $g s p$, rimuovendo un segnale inibitorio, queste mutazioni stimolino la PKA e il signaling ad essa associato. La mutazione nella subunità catalitica (PRKACB) dello stesso complesso proteico in una paziente $\mathrm{CNC}$ acromegalica supporta, in parte, questa ipotesi. D'altra parte, la prevalenza simile di soggetti acromegalici fra pazienti CNC con e senza mutazioni PRKARIA, suggerisce il coinvolgimento di altre vie molecolari [5].

\section{Terapia}

Per nessuno dei trattamenti farmacologici comunemente utilizzati nella terapia degli AI ci sono dati sufficienti nei pazienti CNC per alcun tipo di raccomandazione. Tuttavia, pazienti CNC con acromegalia biochimica senza evidenti lesioni ipofisarie all'imaging sono generalmente trattati con analoghi della somatostatina fino all'eventuale comparsa di un AI.

Per quanto riguarda il trattamento chirurgico, l'adenomectomia selettiva rimane quello d'elezione anche nel CNC. Tuttavia, la presenza di adenomi multipli, comune in questa patologia, associata a ipersecrezione ormonale può richiede- re l'ipofisectomia parziale o totale per ottenere la remissione biochimica [6].

Funding Note Open access funding provided by Università degli Studi di Padova within the CRUI-CARE Agreement.

Conflitto di interesse Gli autori Gianluca Occhi, Daniela Regazzo e Mattia Barbot dichiarano di non avere conflitti di interesse.

Consenso informato Lo studio presentato in questo articolo non ha richiesto sperimentazione umana.

Studi sugli animali Gli autori di questo articolo non hanno eseguito studi sugli animali.

Nota della casa editrice Springer Nature rimane neutrale in riguardo alle rivendicazioni giurisdizionali nelle mappe pubblicate e nelle affiliazioni istituzionali.

Open Access This article is licensed under a Creative Commons Attribution 4.0 International License, which permits use, sharing, adaptation, distribution and reproduction in any medium or format, as long as you give appropriate credit to the original author(s) and the source, provide a link to the Creative Commons licence, and indicate if changes were made. The images or other third party material in this article are included in the article's Creative Commons licence, unless indicated otherwise in a credit line to the material. If material is not included in the article's Creative Commons licence and your intended use is not permitted by statutory regulation or exceeds the permitted use, you will need to obtain permission directly from the copyright holder. To view a copy of this licence, visit http://creativecommons.org/licenses/by/4.0/.

\section{Bibliografia}

1. Stratakis CA, Raygada M (2005) Carney complex. In: Adam MP, Ardinger HH, Pagon RA et al (eds) GeneReviews ${ }^{\circledR}$ [Internet]. University of Washington, Seattle

2. Cuny T, Mac TT, Romanet P et al (2019) Acromegaly in Carney complex. Pituitary 22(5):456-466

3. Kirschner LS (2010) PRKAR1A and the evolution of pituitary tumors. Mol Cell Endocrinol 326(1-2):3-7

4. Pepe S, Korbonits M, Iacovazzo D (2019) Germline and mosaic mutations causing pituitary tumours: genetic and molecular aspects. J Endocrinol 240(2):R21-R45

5. Bertherat J, Horvath A, Groussin L et al (2009) Mutations in regulatory subunit type $1 \mathrm{~A}$ of cyclic adenosine $5^{\prime}$-monophosphatedependent protein kinase (PRKAR1A): phenotype analysis in 353 patients and 80 different genotypes. J Clin Endocrinol Metab 94(6):2085-2091

6. Lonser RR, Mehta GU, Kindzelski BA et al (2017) Surgical management of Carney complex-associated pituitary pathology. Neurosurgery 80(5):780-786 